\title{
PADRÃO ALIMENTAR DE ESTUDANTES UNIVERSITÁRIOS E SUAS IMPLICAÇÕES SOBRE OS FATORES DE RISCO CARDIOVASCULAR
}

\section{FOOD PATTERN OF UNIVERSITY STUDENTS AND THEIR IMPLICATIONS ON CARDIOVASCULAR RISK FACTORS}

\section{ESTÁNDAR DE ALIMENTOS DE ESTUDIANTES UNIVERSITARIOS Y SUS IMPLICACIONES EN FACTORES DE RIESGO CARDIOVASCULAR}

\author{
Andressa Agnolin De Oliveira ${ }^{1}$, Angela Makeli Kososki Dalagnol ${ }^{2}$, Fabiana Brum Haag ${ }^{3}$, Debora Tavares \\ Resende e Silva ${ }^{4}$, Erica de Brito Pitilin ${ }^{5}$, Débora Cristina Fávero ${ }^{6}$
}

Como citar esse artigo: Oliveira AA, Dalagnol AMK, Haag FB, Silva DTR, Pitilin EB, Fávero DC. Padrão alimentar de estudantes universitários e suas implicações sobre os fatores de risco cardiovascular. Rev Enferm Atenção Saúde [Internet]. 2021 [acesso em___];10(2):e202125. doi:10.18554/reas.v10i2.4684

\section{RESUMO}

Objetivo: descrever o padrão alimentar e os fatores de risco cardiovasculares dos estudantes universitários, correlacionando-os ao índice de massa corporal e à pressão arterial sistólica. Métodos: estudo transversal, com 128 estudantes de uma Universidade Federal do Sul do país, que responderam o questionário Guia Alimentar para População Brasileira, houve coleta de medidas antropométricas e pressão arterial sistêmica. Procederam-se as análises: descritiva e correlação de Pearson ( $\mathrm{p}<0,05)$. Resultados: observou-se padrão alimentar insatisfatório com consumo elevado de embutidos $(75,7 \%)$ e carboidratos $(54,6 \%)$, e prática de atividade física abaixo do recomendado $(57,0 \%)$. Verificou-se correlação forte e negativa entre índice de massa corporal e padrão alimentar insatisfatório $(\mathrm{r}=-0,77 ; \mathrm{p}=0,040)$, ou seja, o padrão alimentar insatisfatório relacionou-se ao maior índice de massa corporal. Conclusão: o padrão alimentar insatisfatório é frequente entre os universitários, bem como a inatividade física. Há relação entre o índice de massa corporal e o padrão alimentar de universitários.

Descritores: Estudantes; Padrão Alimentar; Fatores de risco; Doenças cardiovasculares.

\footnotetext{
${ }^{1}$ Universidade Federal da Fronteira Sul - Chapecó/SC. Acadêmica do curso de enfermagem da Universidade Federal da Fronteira Sul - campus Chapecó.

${ }^{2}$ Universidade Federal da Fronteira Sul - Chapecó/SC. Acadêmica do curso de enfermagem da Universidade Federal da Fronteira Sul - campus Chapecó.

${ }^{3}$ Universidade Federal da Fronteira Sul- Chapecó/SC. Mestre em Ciências da Saúde: Cardiologia (IC-FUC). Professora Assistente do Curso de Graduação em Enfermagem da Universidade Federal da Fronteira Sul- Campus Chapecó. Membro do Grupo de Pesquisa Interdisciplinar em Saúde e Cuidado - GEPISC.

${ }^{4}$ Universidade Federal da Fronteira Sul- Chapecó/SC. Doutora em ciências da saúde (UFTM). Professora Coordenadora do Programa de pós graduação em ciências biomédicas e professora adjunta dos cursos de medicina e enfermagem da Universidade Federal da Fronteira Sul - campus Chapecó. Líder do grupo de pesquisa GEPISC. ${ }^{5}$ Universidade Federal da Fronteira Sul- Chapecó/SC. Dra. Doutora em Ciência da Saúde- Universidade Federal de São Paulo (UNIFESP). Professora Assistente do Curso de graduação em Enfermagem Universidade Federal da Fronteira Sul - campus Chapecó. Enfermeira Obstetra. Vice-líder do Grupo de Estudo, Pesquisa Interdisciplinar Saúde e Cuidado (GEPISC)/CNPq.

${ }^{6}$ Universidade de Passo Fundo - Passo Fundo/RS. Enf. Esp. em Cardiologia. Residência Multiprofissional em Cardiologia HCPF/UPF/PMPF. Enfermeira assistente no Hospital de Clínicas de Passo Fundo, Passo Fundo/RS.
} 


\begin{abstract}
Objective: describe the dietary pattern and cardiovascular risk factors of university students and correlate it with body mass index and systolic blood pressure. Methodology: This crosssectional study, with 128 students from a Federal University south of the country, subjected to investigated through a Food Guide questionnaire, and collect anthropometric and systemic blood pressure measures. The following analyzes were performed: descriptive and Pearson's correlation $(p<0,05)$. Results: Low dietary pattern, high consumption of sausages $(75,7 \%)$ and carbohydrates $(54,6 \%)$, predominance of physical activity below the recommended $(57,0 \%)$. It was found strong and negative correlation between body mass index and inadequate dietary pattern $(\mathrm{r}=-0,77 ; \mathrm{p}=0,040)$, that is, the unsatisfactory dietary pattern was related to the higher body mass index. Conclusion: Unsatisfactory eating patterns are common among university students, as well as physical inactivity. There is a relationship between the body mass index and the dietary pattern of university students.
\end{abstract}

Descriptors: Students; Food pattern; Risk factors; Cardiovascular diseases.

\title{
RESUMEN
}

Objetivo: Describir el patrón dietético y los factores de riesgo cardiovascular de los estudiantes universitarios y correlacionarlo con el índice de masa corporal y la presión arterial sistólica. Metodología: Estudio transversal, con 128 estudiantes de la Universidad Federal sur del pais, hecho bajo entrevista a través de cuestionario Guía de Alimentos, y colección de medidas antropométricas, presión arterial sistémica. Los análisis se realizaron: descriptivo y correlación de Pearson ( $\mathrm{p}<0,05)$. Resultados: Bajo patrón dietético, alto consumo de embutidos $(75,7 \%)$ y carbohidratos $(54,6 \%)$, predominio de la actividad física por debajo de lo recomendado $(57,0 \%)$. Fue encontrado correlación fuerte y negativa entre el índice de masa corporal y el patrón dietético inadecuado $(\mathrm{r}=-0,77 ; \mathrm{p}=0,040)$, es decir, el patrón dietético insatisfactorio se relacionó con un indice de masa corporal más alto. Conclusión: Los patrones de alimentación insatisfactorios son comunes entre los estudiantes universitarios, así como la inactividad física. Existe una relación entre el índice de masa corporal y el patrón dietético de los estudiantes universitarios.

Descriptores: Estudiantes; Estándar de alimentos; Factores de riesgo; Enfermedades cardiovasculares.

\section{INTRODUÇÃO}

A cada ano o número de ingressantes na rede superior de ensino aumenta. No ano de 2016, em torno de 3 milhões de pessoas matricularam-se nas universidades Brasileiras. ${ }^{1}$ Nesse cenário, o acesso à universidade é marcado, para os estudantes, como uma fase de mudanças em suas vidas, uma vez que, ao afastar-se do vínculo familiar, passam a assumir novas responsabilidades, tais como cuidar da própria alimentação, pensar em moradia e realizar a administração financeira de sua vida. Diante dessa nova rotina, alimentos práticos e rápidos passam a ser os mais consumidos, ocasionando padrões alimentares menos saudáveis. ${ }^{2}$

O Ministério da Saúde $(\mathrm{MS})^{3}$ entende a alimentação saudável como um direito humano. Nesse sentido, compreende padrão alimentar adequado como aquele que atende às necessidades biológicas e 
sociais dos indivíduos de acordo com as fases da vida. Busatto et al. ${ }^{4}$ relatam em seu estudo que a alimentação possui um significado abrangente, pois envolve escolhas, seleções, ocasiões, rituais e também emoções, além disso, está inserida no contexto da promoção da saúde.

Uma alimentação equilibrada pode prevenir muitas enfermidades, entre elas, as doenças cardiovasculares (DCV), promovendo melhorarias na qualidade de vida. Estudos já comprovam os efeitos de uma dieta saudável e sugerem modelos alimentares capazes de favorecer a proteção cardiovascular, sendo para isso uma das mais citadas a dieta mediterrânea, devido a sua relação com menos eventos cerebrovasculares e cardiovasculares. ${ }^{5}$

A população universitária constituise, em sua maioria, por jovens saudáveis, porém com a presença de alguns fatores de risco cardiovascular (FRCV) intrínsecos ao ambiente acadêmico, com recursos cognitivos de prevenção e mudança de comportamento. Muitas condutas são indicadas e podem ser implementadas dentro do ambiente universitário para um estilo de vida saudável, sendo a dieta uma delas. ${ }^{6}$

O estudo de Framingham ${ }^{7}$ baliza os FRCV tradicionais como tabagismo, obesidade, hipertensão arterial sistêmica (HAS), dislipidemias e diabetes mellitus como fatores que aumentam a probabilidade, em dez anos, para o desenvolvimento de DCV. Outros estudos epidemiológicos elencam diferentes fatores de risco para desenvolver DCV e chamam a atenção para os FRCV não tradicionais, como estilo de vida, estresse e dieta protetora. ${ }^{8}$ A American Heart Association ${ }^{9}$ e a Sociedade Brasileira de Cardiologia ${ }^{8}$ salientam e afirmam que a má alimentação, inatividade física e obesidade são fatores desencadeadores de grande relevância para o desenvolvimento de DCV.

Dessa forma, identifica-se que jovens, em especial os universitários, devido às demandas acadêmicas, requerem atenção e cuidado, com destaque para a implementação de programas com o intuito de melhorar os padrões alimentares, bem como os que visam à prevenção primária de DCV, como incentivo à prática de atividade física e dieta equilibrada, uma vez que se trata de uma população saudável e com inúmeras possibilidades de prevenção e minimização dos FRCV. ${ }^{10}$

Tendo em vista esse cenário, este estudo tem como objetivo descrever o padrão alimentar e os fatores de risco cardiovasculares dos estudantes universitários, correlacionando-os com o índice de massa corporal (IMC) e a pressão arterial sistólica (PAS). 


\section{MÉTODO}

Estudo transversal, realizado entre junho e julho de 2018, com 128 estudantes universitários de uma Universidade Federal do Sul do país. A amostra foi selecionada por conveniência; e a coleta de dados foi realizada por meio do questionário Guia Alimentar para População Brasileira do MS (2014), que contém 14 perguntas. Além disso, usou-se um questionário com informações sociodemográficas, (idade, sexo e nacionalidade), curso de graduação frequentado e meio de transporte utilizado para ir à Universidade.

Também foram aplicadas questões de saúde, entre elas tabagismo, antecedentes familiares com histórico de DCV e prática de atividade física regular, a qual foi medida pelas respostas marcadas (não pratica, pratica pelo menos 30 minutos por dia e pratica 2 a 4 vezes por semana). A classificação em praticantes e não praticantes de atividade física regular baseou-se nas indicações do American College Sports of Medicine (ACMS) ${ }^{12}$, o qual recomenda realizar 30 minutos de atividade física, durante 5 dias da semana, com intensidade moderada - caso a prática seja mais rigorosa, recomenda 20 minutos em 3 dias na semana.

No público-alvo, foram incluídos estudantes matriculados em cursos de períodos integrais, que estavam frequentando entre o terceiro e o nono semestre da graduação, com idade maior ou igual a 18 anos. Foram excluídos aqueles com DCV autodeclarada e os que não autorizaram a verificação de suas medidas antropométricas.

No momento da aplicação dos instrumentos, foram mensuradas a circunferência abdominal (CA) para identificar gordura visceral, sendo CA mulher $\geq 80 \mathrm{~cm}$ e CA homem $\geq 94 \mathrm{~cm}$, seguindo as recomendações de medidas do ACMS. ${ }^{12}$ Também foi verificada a pressão arterial sistêmica (PA), seguindo o método auscultatório padronizado segundo Potter ${ }^{13}$, em que se considerou a PAS $\leq 120 \mathrm{mmHg}$ e a pressão arterial diastólica $(\mathrm{PAD}) \leq 80$ $\mathrm{mmHg}$ como referencial de normalidade. ${ }^{8}$

Para a avaliação antropométrica, foram extraídos parâmetros associados ao risco cardiovascular, como o IMC, o qual avalia o estado nutricional, calculado a partir do peso e altura autorreferidos. Além disso, a classificação foi feita seguindo os parâmetros de normalidade baseados no $\mathrm{MS}^{11}$, sendo eles: IMC $<18,5$ baixo peso, IMC entre 18,5 e $<25 \mathrm{~kg} / \mathrm{m}^{2}$ eutrofia, IMC $\geq 25 \mathrm{e}<30 \mathrm{~kg} / \mathrm{m}^{2}$ sobrepeso, IMC $\geq 30$ $\mathrm{kg} / \mathrm{m}^{2}$ obesidade.

O padrão alimentar foi dicotomizado em satisfatório e insatisfatório. Seguiu-se as recomendações de ingesta diária, sendo para frutas 3 a 5 porções/dia, verduras e legumes 3 a 5 colheres sopa/dia, proteína animal de 1 a 2 
unidades, fatia/dia, carboidratos 6 porções/dia, embutidos 1 porção/semana e água entre 6 a 8 copos/dia, conforme o recomendado pelo Guia Alimentar para População Brasileira. ${ }^{3}$ Nesse sentido, para classificar como satisfatória, considerou-se aqueles que se encontravam com o consumo dentro do recomendado e como insatisfatório, aqueles que não atingiram o consumo mínimo ou ainda passaram do recomendado.

Os dados foram analisados por meio do programa GraphPad Prism versão 7.05. As variáveis contínuas (IMC, PA e CA) foram descritas através de média e desvio padrão. As categóricas (padrão alimentar, atividade física, tabagismo, antecedentes familiares) foram expressas em números absolutos e porcentagem.

Para correlacionar IMC e PAS com padrão alimentar, utilizou-se o coeficiente de correlação de Pearson, levando-se em consideração, para este cálculo, o consumo acima do recomendado de carboidratos e embutidos e o baixo consumo de frutas e verduras. Assim, classificou-se como correlação fraca $(0<\mathrm{r}<0,3)$, moderada $(0,3 \leq$ $r<0,5)$ e forte $(r \geq 0,5) .{ }^{14}$ Os testes foram considerados significativos quando $\mathrm{p} \leq$ 0,05 .

Entretanto, para avaliar o risco cardiovascular, far-se-ia necessário ver as dosagens de colesterol Low Density Lipoprotein (LDL), High Density Lipoprotein (HDL) e triglicérides, bem como seria fundamental um ajuste para a idade padronizada de 60 anos, como já demonstrado por Backer. ${ }^{15}$ Diante disso, os dados foram somente correlacionados com alguns fatores predisponentes de risco cardiovascular, em especial os modificáveis.

O projeto que permitiu a realização da pesquisa foi aprovado com CAAE 69324917.8.0000.5564, sendo coletada assinatura do termo de consentimento livre e esclarecido.

\section{RESULTADOS}

Entre os participantes do atual estudo, verificou-se predomínio do sexo feminino (55,5\%), idade média de 21,6 $(3,2)$ para as mulheres e $22,8(5,0)$ anos nos homens. Outra questão a salientar é que a maioria $(73,4 \%)$ fazia uso do transporte coletivo (Tabela 1). 
Tabela 1 - Dados sociodemográficos e características dos estudantes universitários. Chapecó, SC, Brasil, 2018.

\begin{tabular}{llcll}
\hline Variáveis & $\mathbf{n}$ & $\mathbf{\%}$ & $\mathbf{M}$ & DP \\
\hline Amostra: & 128 & & & \\
Idade: & & & 21,6 & 3,2 \\
$\quad$ Feminino & & & 22,8 & 5,0 \\
$\quad$ Masculino & & & & \\
Sexo: & 71 & 55,5 & & \\
$\quad$ Feminino & 57 & 44,5 & & \\
$\quad$ Masculino & & & \\
Nacionalidade: & 122 & 95,3 & \\
$\quad$ Brasileiro & 6 & 4,7 & \\
$\quad$ Outros & 94 & 73,4 & \\
Meio de transporte: & 27 & 21,1 & & \\
$\quad$ Coletivo & 7 & 5,5 & & \\
$\quad$ Próprio & & & & \\
$\quad$ Escolar & 28 & 21,9 & & \\
Curso de graduação: & 57 & 44,5 & & \\
$\quad$ Administração & 43 & 33,6 & \\
$\quad$ Agronomia & & & \\
$\quad$ Engenharia Ambiental & &
\end{tabular}

Fonte: dados dos autores.

Nota: $\mathrm{M}$ = Média; $\mathrm{DP}$ = desvio padrão.

Em relação à classificação do que a maioria $(71,1 \%)$ consumia o padrão alimentar da amostra, verificou-se preconizado (Tabela 2).

que $14,0 \%$ dos estudantes consumiam frutas Quanto à ingesta de carboidratos e dentro dos valores indicados como embutidos, foi encontrado consumo satisfatórios por dia. No que diz respeito a insatisfatório, ou seja, além do ingesta de proteína animal, encontra-se recomendado $(54,7 \%)$ e $(75,8 \%)$ (Tabela 2$)$. dentro dos padrões recomendados, uma vez 
Tabela 2- Classificação do padrão alimentar dos estudantes universitários, estratificados por grupos alimentares. Chapecó, SC, Brasil, 2018.

\begin{tabular}{lclcc}
\hline $\begin{array}{l}\text { Grupo de } \\
\text { alimentos }\end{array}$ & $\begin{array}{l}\text { *Porções/ } \\
\text { dia }\end{array}$ & $\begin{array}{l}\text { *Critérios adotados } \\
\text { para a adequação }\end{array}$ & $\begin{array}{c}\text { Classificação } \\
\text { alimentação }\end{array}$ & da \\
& & & $\begin{array}{c}\text { Satisfatório } \\
\mathbf{n}(\%)\end{array}$ & $\begin{array}{c}\text { Insatisfatório n } \\
(\%)\end{array}$ \\
Frutas & $3-5$ & $\geq 3$ porções/dia & $18(14,0)$ & $110(85,9)$ \\
Verduras/legumes & $3-5$ & $\geq \quad 5 \quad$ colheres, & $59(46,1)$ & $69(53,9)$ \\
Proteína animal & $1-2$ & $\begin{array}{l}1-2 \text { unidade, fatia/ } \\
\text { dia }\end{array}$ & $91(71,1)$ & $37(28,9)$ \\
Carboidrato & 6 & 6 porções/dia & $58(45,3)$ & $70(54,7)$ \\
Embutidos & 1 & $\begin{array}{l}1 \text { porção/ } \\
\text { Semana }\end{array}$ & $31(24,2)$ & $97(75,8)$ \\
Água & $6-8$ copos/dia & $53(41,4)$ & $75(58,6)$ \\
\hline
\end{tabular}

Fonte: dados dos autores.

Nota: *Guia Alimentar para População Brasileira³.

Identificou-se que $32,0 \%$ dos Sobre o consumo de gorduras estudantes consumiam doces de 2 a 4 vezes (Figura 1 B), constatou-se que a maioria por semana; e 6,3\% todos os dias, sendo $\quad(64,8 \%)$ não consumia gordura aparente na este resultado satisfatório (Figura $1 \mathrm{~A}$ ). carne. Ainda, 3,9\% dos estudantes não consumiam carne vermelha ou frango.

Figura 1 - A) Consumo de doces e B) Consumo de gorduras. Chapecó, Santa Catarina, Brasil, 2018

A) Frequência do consumo de doces, refrigerantes e sucos industrializados pelos universitários

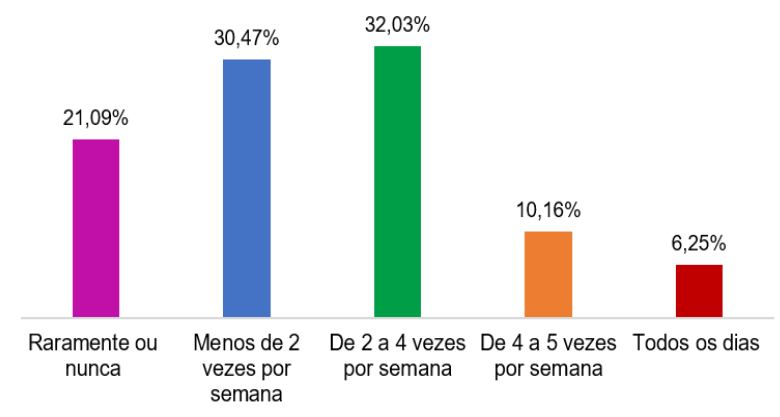

B) Consumo de gorduras aparente na carne e gordura mais utilizada para cozinhar os alimentos.

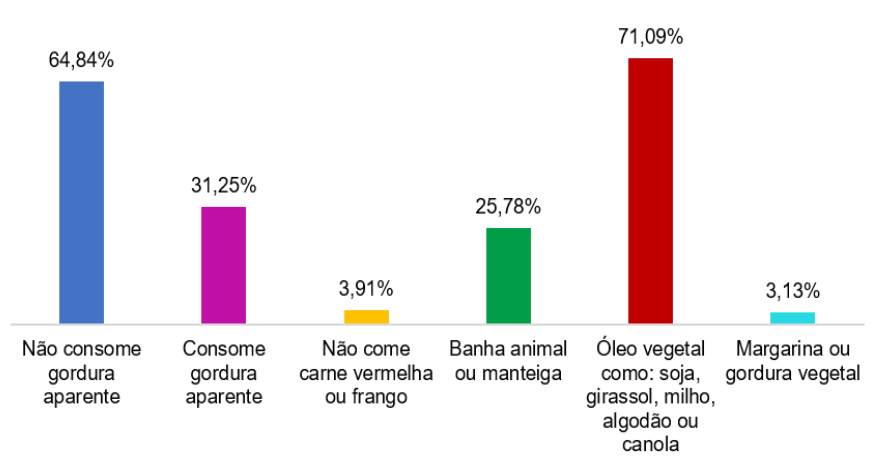

Fonte: dados dos autores. 
No que se refere aos resultados dos FRCV, identificou-se que, 57,3\% dos estudantes não praticam atividade física regular (Tabela 3).

Verificou-se que a maioria dos estudantes do sexo feminino $(69,0 \%)$ e masculino $(94,7 \%)$ estava com a CA adequada (Tabela 3), contudo, destaca-se o percentual inferior entre as mulheres.

Constatou-se que $17,9 \%$ e $0,8 \%$ dos estudantes estavam com a PAS e a PAD alteradas, respectivamente (Tabela 3). A média da PAS foi de 111,6 (14,5); e a PAD de $71,2(8,7)$ o que demonstrou resultados dentro dos valores normais.

Maior percentual $(72,6 \%)$ foi classificado como eutrófico. Entretanto, destaca-se que $16,4 \%$ dos estudantes tinham sobrepeso. A média de IMC na amostra foi de $23,8 \mathrm{~kg} / \mathrm{m}^{2}$ com desvio padrão de 3,6. 
Tabela 3 - Fatores de risco cardiovascular dos estudantes universitários. Chapecó, SC, Brasil, 2018.

\begin{tabular}{|c|c|c|c|}
\hline Variável & $\mathbf{n}$ & $\%$ & M (DP) \\
\hline \multicolumn{4}{|l|}{ - Atividade física: } \\
\hline Não pratica atividade física regular & 73 & 57,0 & \\
\hline Pratica pelo menos $30 \mathrm{~min}$. por dia & 22 & 17,2 & \\
\hline Pratica de 2 a 4 vezes por semana & 33 & 25,8 & \\
\hline \multicolumn{4}{|l|}{ - Antecedentes familiares: } \\
\hline Sim & 52 & 40,6 & \\
\hline Não & 76 & 59,4 & \\
\hline \multicolumn{4}{|l|}{ - Tabagismo } \\
\hline Fumantes: & 6 & 4,7 & \\
\hline Não fumantes & 122 & 95,3 & \\
\hline $\begin{array}{l}\text {-Circunferência abdominal: } \\
\text { Acima do recomendado: }\end{array}$ & & & $78,8 \quad(9,2)$ \\
\hline Feminino: & 22 & 17,2 & \\
\hline Masculino: & 3 & 2,3 & \\
\hline \multicolumn{4}{|l|}{-Pressão arterial Sistêmica: } \\
\hline Sistólica: & & & $111,6(14,5)$ \\
\hline Diastólica: & & & $71,2 \quad(8,7)$ \\
\hline \multicolumn{4}{|l|}{ Acima do recomendado: } \\
\hline Sistólica: & 23 & 17,9 & \\
\hline Diastólica: & 1 & 0,8 & \\
\hline - Índice de massa corporal: & & & $23,8 \quad(3,6)$ \\
\hline Baixo peso: & 4 & 3,1 & \\
\hline Eutrofia: & 92 & 72,6 & \\
\hline Sobrepeso: & 22 & 16,4 & \\
\hline Obesidade: & 10 & 7,8 & \\
\hline
\end{tabular}

Fonte: dados dos autores.

Nota: $\mathrm{M}(\mathrm{DP})$ = média (desvio padrão).

Por meio dos dados apresentados na

Tabela 4, é possível identificar correlação significativa entre o IMC e o padrão alimentar, sendo esta inversamente proporcional, indicando, na amostra estudada, que o padrão alimentar insatisfatório relaciona-se ao maior IMC, $\operatorname{com} \mathrm{p}=0,040$. 
Tabela 4 - Correlação entre IMC, PAS e padrão alimentar. Chapecó, SC, Brasil, 2018.

\begin{tabular}{ccc}
\hline Variável & $* \mathbf{r}$. & $* * \mathbf{p}$. \\
\hline IMC x Padrão alimentar & $-0,77$ & $\mathrm{p}=0,040$ \\
PAS x Padrão alimentar & $-0,34$ & $\mathrm{p}=0,09$
\end{tabular}

Fonte: dados dos autores.

Nota: *r (correlação de Pearson); ** ( $<<0,05)$.

\section{DISCUSSÃO}

Analisando os resultados descritos, percebe-se que estão alinhados com a profusa base de evidências sobre padrão alimentar em jovens e FRCV. Também foram estabelecidos alguns indícios de FRCV presentes na amostra, como atividade física regular abaixo do recomendado e padrão alimentar insatisfatório em mais de $50 \%$ dos sujeitos em todos os grupos de alimentos (Frutas, verduras, carboidratos, embutidos e água), exceto no item proteína animal, já que a maioria consome as quantidades recomendadas.

No grupo estudado, identificou-se que os jovens são os que mais frequentam a universidade e que houve prevalência do sexo feminino, embora os cursos de agronomia e engenharia ambiental tenham predominância histórica do sexo masculino, resultado esse que está em conformidade com os dados do censo da educação superior de $2016^{1}$, segundo os quais a maioria dos universitários no Brasil atualmente é mulher.
O meio de transporte mais utilizado foi o coletivo, sinalizando que a maioria dos universitários não possuía carro próprio, instigando estes a caminhar até os pontos de ônibus, dentro e fora do campus universitário. Contudo, como destacado pelo $\mathrm{ACMS}^{12}$, para que seja considerada atividade física regular, deve ser realizada com um tempo mínimo de 150 minutos por semana.

Este estudo apontou que os estudantes universitários ingerem com menor frequência diária alimentos considerados de proteção para DCV, como frutas e verduras. Já nesse público aqueles alimentos considerados de risco como os embutidos e os carboidratos têm consumo acima do preconizado pelo Guia Alimentar para a População Brasileira. ${ }^{3}$

Para uma alimentação ser considerada adequada, ou seja, saudável ela precisa conter a melhor combinação de nutrientes. Segundo o $\mathrm{MS}^{3}$, o consumo adequado de frutas e verduras está relacionado ao menor risco de desenvolvimento de doenças crônicas não transmissíveis (DCNT) e à manutenção do 
peso adequado. Igualmente, a pesquisa de Santos et al. ${ }^{2}$ destacou em seus resultados baixa ingesta de frutas e verduras entre os estudantes. É oportuno salientar que alimentação rica em embutidos e pobre em frutas e verduras está entre os principais fatores para o desenvolvimento de DCNT e DCV. ${ }^{7,9}$

Quanto ao consumo de proteína animal, o resultado apresentou ingestão satisfatória. Destaca-se que o consumo de proteína animal como carnes, ovos e peixes é importante devido ao seu alto valor biológico e grande quantidade de proteínas funcionais. Entretanto, o consumo elevado predispõe a um aumento no desenvolvimento de DCV e a alguns tipos de câncer. ${ }^{3}$

Os carboidratos constituem a maior parte da alimentação humana, sendo fonte de energia e vitaminas, essenciais ao funcionamento e metabolismo celular. No entanto, o consumo excessivo aumenta o risco de obesidade e de outras DCNT, bem como causa repercussões no sistema cardiovascular. ${ }^{3}$

Uma semelhança foi encontrada no estudo de Loureiro ${ }^{10}$, no qual o consumo de alimentos como os embutidos aumentou consideravelmente, tanto entre os universitários como na população brasileira. Esses alimentos possuem altas concentrações de gordura e sódio, e o consumo elevado vem sendo apontado como um dos principais fatores de risco de DCNT e de câncer em todo o mundo. ${ }^{3}$

Sobre o consumo de água, observou-se que entre os universitários a ingesta é insatisfatória. Seu uso é fundamental para manutenção da vida, a quantidade que deve ser tomada diariamente varia de indivíduo para indivíduo e conforme o tipo de alimentação, mas a maioria das recomendações indicam que um adulto saudável deve beber um mínimo de 2 litros de água por dia. ${ }^{3}$

Quanto ao consumo de doces, os resultados corroboram com os achados de Santos et al. ${ }^{2}$ que também identificaram que o uso entre universitários é esporádico. Ressalta-se que a ingestão acima do indicado contribui como FRCV por manter a glicemia em níveis elevados. Além disso, está relacionado com DCNT como diabetes mellitus e obesidade. Sendo que estes alimentos poderiam ser substituídos por frutas, principalmente entre os estudantes. ${ }^{3}$

Observou-se alimentação adequado de gorduras e constatou-se que grande parte da amostra não come a gordura aparente na carne. A I Diretriz sobre o consumo de gorduras e saúde cardiovascular ${ }^{16}$ destaca que: "[...] ao se preparar quaisquer tipos de carnes, é necessário que se remova a gordura aparente e a pele (aves), pois a gordura penetra no interior da carne durante o preparo". Verificou-se também o uso de gordura para o cozimento dos alimentos. 
Segundo o $\mathrm{MS}^{3}$, a gordura mais indicada é a de origem vegetal como os óleos, em concordância com o observado nesta amostra.

Após análise dos resultados, denotase que a alimentação dos universitários é insatisfatória, pois, dos 9 grupos alimentares avaliados, identificou-se que em 5 deles o consumo não se encontra dentro dos padrões recomendados. Esse resultado é atribuído às modificações no padrão dietético corriqueiro durante $o$ período universitário, bem como à mudança de cidade ou de Estado, o que indica que a cultura local pode ser um fator significativo a ser considerado. ${ }^{16}$

Em sintonia com este estudo, a pesquisa de Loureiro $^{10}$ afirma que a alimentação dos estudantes de diversos países é considerada inadequada, visto que, entre eles, é elevado o consumo de alimentos processados e baixa a ingestão de alimentos saudáveis.

Além do padrão alimentar, o estudo identificou outros FRCV, entre eles o nível de atividade física, tabagismo, IMC, CA e PA, os quais, se sustentados permanentemente, podem precipitar ou causar DCV e cerebrovascular, bem como representar alto custo em assistência à saúde. ${ }^{17}$

Alguns estudos ${ }^{10,17}$ destacam que os universitários possuem elevado risco de vir a desenvolver DCV devido ao fato de negligenciarem hábitos saudáveis de vida em detrimento das demandas acadêmicas. Em relação à atividade física, fator modificável, observou-se que a prática regular foi abaixo do mínimo recomendado pelo ACMS. ${ }^{12}$ Esses achados corroboram com a pesquisa de Santos et al. ${ }^{2}$ no qual a maioria dos indivíduos $(50,8 \%)$ não pratica atividade física regular, e 34,2\% referiram praticar entre 1 a 3 vezes por semana. Outras análises ${ }^{2,10}$ relacionam o baixo nível de atividade física com a falta de tempo, motivação, apoio social e a distância entre os domicílios e espaços destinados à prática de exercício físico.

A presente pesquisa identificou que parte dos universitários possuíam antecedentes familiares diretos de DCV. O estudo de Gomides et al. ${ }^{18}$ destaca que os fatores hereditariedade e a baixa prática de atividade física contribuem para o risco cardiovascular e que estes estão entre os fatores mais prevalentes de riscos em estudantes.

Quanto ao tabagismo, 4,8\% fumam, sendo um resultado esperado e positivo. Isso porque na maioria das pesquisas com universitários, principalmente as realizadas nos últimos dez anos, o uso de tabaco é sempre baixo ou irrelevante. ${ }^{2}$

A CA é outro parâmetro que atua como complemento para o diagnóstico nutricional pois, mostra a distribuição do tecido adiposo no corpo, quando esse tecido 
se concentra na região central/abdômen tem relação direta com a morbimortalidade. ${ }^{11}$ Salienta-se que foram obtidos resultados dentro dos valores de referências de normalidade. ${ }^{12}$ Já em relação à $\mathrm{PA}$, esta amostra não apresentou alterações significativas. Em contrapartida, o estudo de Ofori et al. ${ }^{18}$ destacou que a ocorrência de PAS (45\%) e PAD $(32,5 \%)$ foi elevada entre os estudantes.

Um dado interessante desta pesquisa foi que, ainda que os estudantes universitários apresentem índice insatisfatório de alimentação, eles mantêm em sua maioria IMC normal. Também no estudo de Marconato et a ${ }^{19}$ a média do IMC identificada foi de 21,8 kg/m2, apesar de padrões alimentares e de atividade física serem abaixo do mínimo recomendado. Este fato pode ser devido aos universitários comporem, em sua maioria, um público jovem, mas que se os maus hábitos forem mantidos, estes podem acarretar em aumento do IMC.

A correlação entre IMC e padrão alimentar foi forte e inversa (negativamente), apontando coerência com demais estudos da área ${ }^{19,20}$ e demonstrando que à medida que aumenta $\mathrm{o} I \mathrm{MC}$, piora $\mathrm{o}$ padrão alimentar. Quanto à PAS e ao padrão alimentar, não houve correlação significativa, talvez por tratar-se de uma amostra jovem e saudável, com PA e IMC, em sua maioria, dentro dos níveis considerados normais.

Sabe-se que a principal etiologia das DCV é aterosclerose, que tem seu início na infância. Dessa forma, é reconhecido que a prevenção cardiovascular deve começar precocemente, com ênfase nos fatores modificáveis, e que o ambiente universitário possui condições estruturais e organizacionais capazes de acolher e promover ações específicas de educações em saúde, como adequação dos restaurantes universitários aos padrões de alimentação saudável, orientações individuais ou em grupos, bem como uso das mídias sociais para disseminação de conhecimento, contribuindo para um espaço mais saudável e proporcionando a formação de indivíduos conscientes da importância da alimentação saudável e da prática regular de atividade física para seu bem-estar. ${ }^{20}$

\section{CONCLUSÕES}

Os padrões alimentares dos estudantes são insatisfatórios e há presença de FRCV, como a prática de atividade física abaixo do mínimo recomendado. Identificou-se que os estudantes universitários apresentaram baixo consumo de frutas, verduras, legumes e alto consumo de carboidratos e embutidos, fatores que, por meio de ações em saúde, podem ser revertidos a fim de melhorar a qualidade de vida. 
Ressalta-se que o tempo de exposição aos fatores preditivos de DCV demarca a possibilidade de intervenção prévia a maiores agravos na saúde, demonstrando a necessidade de medidas educativas e preventivas de saúde, pois a universidade é um ambiente de produção e transferência de saberes e consequentemente um ambiente para promoção da saúde.

A principal limitação desta pesquisa refere-se ao peso e altura autorreferidos. Sublinha-se, ainda, a importância de mais estudos nesta linha e em outras como longitudinal e multicêntrica, pois, por meio de seus resultados, é possível identificar, formular e implementar ações de intervenção, prevenção e promoção de saúde no ambiente universitário.

Finaliza-se reforçando que as universidades se constituem num espaço fértil para a promoção de estratégias de educação em saúde e estilos de vida saudáveis, com possibilidade de realização de atividades voltadas para a redução do excesso de peso e obesidade, incentivo à prática de atividade física e à alimentação saudável para que haja consequentemente a diminuição de FRCV.

\section{REFERÊNCIAS}

1. Brasil. Instituto Nacional de Estudos e Pesquisas Educacionais Anísio Teixeira. MEC e Inep divulgam dados do Censo da Educação Superior 2016 - 2017. [internet].
2017 [citado em 06 out. 2018]; Disponível em: http://portal.inep.gov.br/artigo//asset_publisher/B4AQV9zFY7Bv/content /mec-e-inep-divulgam-dados-do-censo-daeducacao-superior-2016/21206

2. Santos AKGV, Reis CC, Chaud DMA, Morimoto JM. Qualidade de vida e alimentação de estudantes universitários que moram na região central de São Paulo sem a presença dos pais ou responsáveis. Rev Simbio-Logias [internet]. Botucatu 2014; 7 (10);76-99 [citado em 28 ago. 2018]; Disponível em: http://www.ibb.unesp.br/Home/Departame ntos/Educacao/Simbio-

Logias/qualidade_de_vida_alimentacao_de _estudantes.pdf

3. Brasil. Ministério da Saúde. Secretaria de Atenção à Saúde. Guia alimentar para a população brasileira. Departamento de Atenção Básica. [publicação online] Brasília, 2014; p. 156 [citado em 04 out. 2018]; Disponível em:

http://portalarquivos.saude.gov.br/images/p df/2014/novembro/05/Guia-Alimentarpara-a-pop-brasiliera-Miolo-PDFInternet.pdf

4. Busato MA, Pedrolo C, Gallina LS, Rosa L. Ambiente e alimentação saudável: percepções e práticas de estudantes universitários. Semina: Ciências Biológicas e da Saúde, Londrina 2015; 2 (36);75-84 [citado em 08 out. 2018]; Disponível: doi: 10.5433/16790367.2015v36n2p75

5. Noite A, Pinto J, Freitas CP, Melo C, Albuquerque A, Teixeir M, Bastos JM. Efeitos da dieta mediterrânica e exercício físico em indivíduos com doença arterial coronária. Revista Portuguesa de Cardiologia, [internet] [s.1.], 2015; 34 (11): 655-664 [citado em 20 out. 2018];

Disponível em: doi: http://dx.doi.org/10.1016/j.repc.2015.05.00 4.

6. Leppink EW, Odlaug BL, Lust K.

Christenson G, Grant JE. The Young and the Stressed: Stress, Impulse Control, and Health in College Students. J Nerv Ment Dis 2016; 204(12):931-938 [citado em 15 
out. 2019]; Disponível em: doi: http://dx.doi.org/10.1097/nmd.0000000000 000586.

7. Study, Framingham Heart. The Framingham Heart Study is a project of the National Heart, Lung, \& Blood Institute \& Boston University. FraminghamMassachusetts- EUA. 1948-2018 [citado em 27 out. 2018]; Disponível em: https://www.framinghamheartstudy.org/ 8. $7^{\text {a }}$ diretriz brasileira de hipertensão arterial. Arquivo Brasileiro Cardiologia. Revista da sociedade brasileira de cardiologia, Rio de Janeiro, 2016; 107 (3): 1-103 [citado em 08 out. 2018]; Disponível em:

http://publicacoes.cardiol.br/2014/diretrize s/2016/05_HIPERTENSAO_ARTERIAL. pdf

9. Levine GN, Lange RA, BaireyMerz CN, et al. Cardiovascular risk reduction: A scientific statement from the American heart association. J Am Heart Assoc. 2017; 6(10): e002218; [citado em: 15 nov. 2018]; Disponível em: http://dx.doi.org/10.1161/jaha.117.002218. 10. Loureiro MP. Estado nutricional e hábitos alimentares de universitários. Segurança Alimentar e Nutricional, Campinas, 2016; 2(23):955-972 [citado em 08 out. 2018]; Disponível em: doi.org/10.20396/san.v23i2.8647612 11. Brasil. Ministério da Saúde. Secretaria de Atenção à Saúde. Departamento e Atenção Básica. Orientações para a coleta e análise de dados antropométricos em serviços de saúde: Norma Técnica do Sistema de Vigilância Alimentar e Nutricional - SISVAN. Secretaria de Atenção à Saúde. Departamento de Atenção Básica. [internet] Brasília, 2011; p. 76 [citado em 16 set. 2018]; Disponível em:

http://bvsms.saude.gov.br/bvs/publicacoes/ orientacoes_coleta_analise_dados_antropo metricos.pdf

12. ACMS, American College of Sports

Medicine position statement on the recommended quantity and quality of exercise for developing and maintaining fitness in healthy adults. Med Sci Sports. Indianapolis. 1998; 30(6): 975-991 [citado em: 09 nov. 2018]; Disponível em: doi.org/10.1097/00005768-19980600000032

13. Potter P, Perry AG, Stockert P, Hall A. Fundamentos de Enfermagem. $9^{a}$ ed. Rio de Janeiro: Elsevier, 2018.

14. Cohen J. (1988), Statistical power analysis for the behavioral sciences. Hillsdale, NJ, Erlbaum.

15. Backer G, Ambrosionie E, Johnsena BK, Brotonsh C , Cifkovae R, Dallongeville J, et al. European guidelines on cardiovascular disease prevention in clinical practice Third Joint Task Force of European and other Societies on Cardiovascular Disease Prevention in Clinical Practice (constituted by representatives of eight societies and by invited experts). Oxford University Press (OUP). European Heart Journal, [s.1.], 2003; 24 (17): 1601-1610 [citado em: 13 abr. 2019]; Disponível: Doi: doi.org/10.1016/s0195-668x(03)00347-6.

16. I Diretriz sobre o Consumo de Gorduras e Saúde Cardiovascular. Arquivo Brasileiro Cardiologia. Revista da sociedade brasileira de cardiologia, Rio de Janeiro 2013; 100 (1): 1-49. [citado em: 15 nov. 2018]. Disponível em:

http://publicacoes.cardiol.br/consenso/201

3/Diretriz_Gorduras.pdf

17. Ofori EK, Inttiful FD, Asante M, Asare GA, Adjei PK, Dadzie RKS, et al . Prevalence of cardiovascular disease risk factors among students of a tertiary institution in Ghana. Food Science \& Nutrition, Estados Unidos, 2018; 6(2):381387 [citado em 18 out. 2018]; Disponível: doi: doi.org/ 10.1002/fsn3.565

18. Gomides PHG, Moreira OC, Oliveira RAR, Filho MLM, Matos DG, Oliveira CEP. Determinação do risco coronariano em estudantes de educação física de uma universidade pública do estado de Minas Gerais como estratégia de avaliação préparticipação. Revista Brasileira de Prescrição e Fisiologia do Exercício. São Paulo, 2014; 8(48): 565-570 [citado em 16 
set. 2018]; Disponível em:

http://www.rbpfex.com.br/index.php/rbpfe

x/article/download/655/627

19. Marconato, MSF; Silva, GMM;

Frasson, TZ. Hábito alimentar de

universitários iniciantes e concluintes do

curso de Nutrição de uma Universidade do

interior Paulista. Revista Brasileira de

Obesidade, Nutrição e Emagrecimento,

São Paulo, 2016; 10 (58):180-188 [citado

em 15 jul. 2020]; Disponível em:

https://dialnet.unirioja.es/servlet/articulo?c

odigo $=5580207$.

20. Macedo TT, Carneiro MF, Da Silva

CGP, Brito TJR, Pereira PP. Perfil

alimentar, clínico e padrão de atividade

física em ingressantes universitários de

enfermagem. Rev Cubana Enfermagem

[Internet]. 2019; 35(1) [citado 19 Jul

2020]; Disponível

em: http://www.revenfermeria.sld.cu/index

.php/enf/article/view/1785

RECEBIDO: $18 / 06 / 2020$

APROVADO: $08 / 01 / 2021$

PUBLICADO: 09/2021 